\title{
Power and Rate Control with Outage Constraints in CDMA Wireless Networks
}

\author{
C. Fischione, M. Butussi, K. H. Johansson, and M. D’Angelo
}

\begin{abstract}
A radio power control strategy to achieve maximum throughput for the up-link of CDMA wireless systems with variable spreading factor is investigated. The system model includes slow and fast fading, rake receiver, and multi-access interference caused by users with heterogeneous data sources. The quality of the communication is expressed in terms of outage probability, while the throughput is defined as the sum of the users' transmit rates. The outage probability is accounted for by resorting to a lognormal approximation. A mixed integer-real optimization problem $\mathcal{P}_{1}$, where the objective function is the throughput under outage probability constraints, is investigated. Problem $\mathcal{P}_{1}$ is solved in two steps: firstly, we propose a modified problem $\mathcal{P}_{2}$ to provide feasible solutions, and then the optimal solution is obtained with an efficient branch-and-bound search. Numerical results are presented and discussed to assess the validity of our approach.
\end{abstract}

Index Terms-CDMA, outage, distributed computation, combinatorial optimization, branch-and-bound search.

\section{INTRODUCTION}

A $\mathrm{N}$ optimization problem to maximize the up-link throughput achievable in CDMA wireless systems by radio power allocation is investigated. We express quality of service constraints by outage probability for any user, which is an important measure for delay-limited transmissions [1].

The problem of power control under outage constraints dates back at least to the work presented in [2]. In [3], the authors consider a problem under outage probability constraints in Rayleigh fading channels. This approach has later been further developed in [4], where the channel is modeled with lognormal fading, and the outage probability is approximated by a Gaussian distribution. Both in [3] and [4], it is shown that the power minimization under outage constraints can be cast as a geometric program. An interesting approach to the power control with outage constraints is investigated in [5], [6], where an iterative method is presented for some distinct cases of slow and fast fading. The outage constraints have

Paper approved by D. I. Kim, the Editor for Spread Spectrum Transmission and Access of the IEEE Communications Society. Manuscript received June 18, 2007; revised July 9, 2008.

C. Fischione and K. H. Johansson are with the ACCESS Linnaeus Center, Electrical Engineering, Royal Institute of Technology, Stockholm, Sweden (e-mail: \{carlofi, kallej\}@ee.kth.se).

M. Butussi was with the University of Padova, Italy, when this work was done (e-mail: bokassa@dei.unipd.it).

M. D'Angelo is with the University of L'Aquila and PARADES GEIE, Italy (e-mail: mdangelo@parades.rm.cnr.it).

This work was done in the framework of the HYCON Network of Excellence, contract number FP6-IST-511368. The work is also partially funded by the Swedish Foundation for Strategic Research and Swedish Research Council. M. D'Angelo acknowledges the support of Fondazione Ferdinando Filauro, Italy.

Part of this work was presented at IEEE ICC 2007.

Digital Object Identifier 10.1109/TCOMM.2009.08.070288 been relaxed with an upper bound provided by the Jensen's inequality.

In this paper we investigate a mixed integer-real optimization problem which is different from those in the existing contributions from the literature. The constraints on the outage are expressed by a lognormal approximation studied in [7], which allows us to solve the problem even for low outage probabilities. Our contribution is original, for example, compared to the interesting works [3] - [5], because they do not include a detailed model of the Multi Access Interference (MAI) and the source data, while we adopt a general model of the SINR, which is representative of mixed slow and fast fading. We propose a new method to solve the rate maximization problem, which is based on branch-and-bound search.

\section{System Description AND PROBlem Formulation}

Consider a system scenario where $K$ mobile users transmit to a base station. Each user $i=1, \ldots, K$ is associated with a traffic source type (voice, video, data, etc.), a level of transmission power $P_{i}$, and a chip time $T_{c}$. Let $h_{i}$ be the channel gain for user $i$. We adopt the Lee and Yeh multiplicative model [8, pag. 91]: $h_{i}=l_{i} z_{i} \Omega_{i}$, where $l_{i}$ is the path loss, $z_{i}$ is the power of the fast fading, and $\Omega_{i}$ is the power of the shadowing. A Rayleigh distribution for the fast fading is assumed. For any $i \neq j, z_{i}$ and $z_{j}$ are statistically independent. Let $\Omega_{i}=\exp \left(\xi_{i}\right)$, where $\xi_{i}$ is a Gaussian random variable having zero average and standard deviation in linear units $\sigma_{\xi_{i}}$. Let the binary random variable $\nu_{i}$ be the activity status (on/off) of the source. The probability mass function is such that $\operatorname{Pr}\left[\nu_{i}=1\right]=\alpha_{i}$ and $\operatorname{Pr}\left[\nu_{i}=0\right]=1-\alpha_{i}$, where $\alpha_{i}$ is the activity factor of source $i$. Let $\mathbf{h}=\left[h_{1}, \ldots, h_{K}\right]^{T}$ and $\boldsymbol{\nu}=\left[\nu_{1}, \ldots, \nu_{K}\right]^{T}$. Independence is assumed between any pair of random variables of the vectors $h$ and $\nu$. The model of the physical layer for the up-link of a single-cell asynchronous binary phase shift keying DS/CDMA system is summarized by the following expression of the SINR [9]:

$$
\operatorname{SINR}_{i}(\mathbf{h}, \boldsymbol{\nu})=\frac{P_{i} h_{i}}{\frac{N_{0}}{2 T_{i}}+\sum_{\substack{j=1 \\ j \neq i}}^{K} \frac{P_{j}}{G_{i}} h_{j} \nu_{j}},
$$

Observe that this SINR model takes into account also a rake receiver with Maximal Ratio Combining [10].

The rate of user $i, i=1, \ldots, K$, is modeled as $R_{i}=R_{i 0} n_{i}$, where $R_{i 0}=1 / T_{i 0}$ is the basic rate with basic bit time $T_{i 0} . n_{i}$ is an integer denoting the assigned rate, which is a power of two due to the spreading codes [8]. The spreading factors are expressed by $G_{i}=G_{i 0} / n_{i}$, where $G_{i 0}=T_{i 0} / T_{c}$ corresponds to the basic rate $R_{i 0}$. We assume that the radio power of a user is expressed as $P_{i}=p_{i} n_{i}$, where $p_{i}$ is 
the power at the basic rate $R_{i 0}$ (i.e., when $n_{i}=1$ ). This power model is motivated by the fact that users requiring larger rates need larger transmit powers, as shown in [9]. This model has been adopted also in [11] (and references therein), where it has been observed that it has the advantage of keeping the bit energy to noise ratio constant with respect to variations of $n_{i}$. Let $\mathbf{p}=\left[p_{1}, \ldots, p_{K}\right]^{T}, \mathbf{p}_{-\mathbf{i}}=$ $\left[p_{1}, p_{2}, \ldots, p_{i-1}, p_{i+1} \ldots p_{K}\right]^{T}, \mathbf{n}=\left[n_{1}, n_{2}, \ldots, n_{K}\right]^{T}$ and $\mathbf{n}_{-\mathbf{i}}=\left[n_{1}, n_{2}, \ldots, n_{i-1}, n_{i+1}, \ldots n_{K}\right]^{T}$.

The objective function of the rate maximization problem is the sum of the users' rates, and the constraints are bounds on the maximum value for the outage probability, transmission powers and rates:

$$
\begin{aligned}
& \mathcal{P}: \quad \max _{\mathbf{p}, \mathbf{n}} \mathbb{1}^{T} \mathbf{n} \\
& \text { s.t. } \quad \operatorname{Pr}\left[\operatorname{SINR}_{i}(\mathbf{h}, \boldsymbol{\nu})<\gamma_{i}\right] \leq O_{i}, \quad \forall i=1, \ldots, K \\
& \mathbf{p}^{T}(\mathbf{n} \circ \mathbb{E}[\mathbf{h}]) \leq P_{T}, \\
& p_{i 0} \leq p_{i} \leq p_{\text {imax }}, \quad \forall i=1, \ldots, K \\
& 1 \leq n_{i} \leq G_{i 0}, \quad n_{i} \in \mathcal{N}, \quad \forall i=1, \ldots, K
\end{aligned}
$$

where $\mathbb{1}^{T}=\left[\begin{array}{ll}1 & 1 \ldots 1\end{array}\right] \in \mathbb{R}^{K}$, and $\mathbf{l}^{T}=\left[\begin{array}{ll}l_{1} & l_{2} \ldots l_{K}\end{array}\right]$. The decision variables are the powers $\mathbf{p}$ and rates n. $O_{i}$ is the maximum outage probability that is allowed for user $i$ with respect to the threshold $\gamma_{i}$. The constraint $\mathbf{p}^{T}(\mathbf{n} \circ \mathbb{E}[\mathbf{h}])=$ $\sum_{i=1}^{K} n_{i} p_{i} l_{i} \exp \left(\sigma_{\xi_{i}}^{2} / 2\right) \leq P_{T}$ is due to the fact that the antenna of the base station can receive only a maximum amount of power without distorting the signal [9]. For obvious physical reasons, powers cannot be smaller than a given value $p_{i 0}$, nor larger than a maximum power $p_{i \max }$. The rate constraint follows from the fact that $G_{i}=G_{i 0} / n_{i} \geq 1$. Finally, the set of rates, $\mathcal{N}$, contains only power of two elements.

Remark 1: We assume that an admission control policy is used (see e.g. [12]), so that all $K$ users can be accommodated in the system while transmitting at least with the minimum power and rate while satisfying all constraints of $\mathcal{P}$. Consequently, Problem $\mathcal{P}$ is feasible.

In the next section, we propose a method to solve Problem $\mathcal{P}$ efficiently.

\section{Problem Solution}

\section{A. Outage Probability}

Knowledge of the outage probability is required to express the first constraint in Problem $\mathcal{P}$. Since a closed form expression for such a probability is unknown, we adopt a lognormal approximation of the SINR pdf, as proposed in [7]. Using such an approximation, the derivation of the outage probability can be easily accomplished, and it can be shown that the constraint is expressed as

$$
\begin{aligned}
p_{i} \geq \gamma_{i} I_{i}\left(\mathbf{n}_{-i}, \mathbf{p}_{-i}\right)= & \gamma_{i} \exp \left(-q_{i} \sqrt{\zeta(2,1) \sigma_{X_{i}}^{2}\left(\mathbf{n}_{-i}, \mathbf{p}_{-i}\right)}\right. \\
& \left.+\mu_{X_{i}}\left(\mathbf{n}_{-i}, \mathbf{p}\right)+\ln p_{i}-\psi(1)\right),
\end{aligned}
$$

where $q_{i}=Q^{-1}\left(1-O_{i}\right), Q(x)=1 / \sqrt{2 \pi} \int_{x}^{\infty} e^{-t^{2} / 2} d t$ is the complementary standard Gaussian distribution, and $\mu_{X_{i}}\left(\mathbf{n}_{-i}, \mathbf{p}\right)=2 \ln M_{i}^{(1)}\left(\mathbf{n}_{-i}, \mathbf{p}\right)-\frac{1}{2} \ln M_{i}^{(2)}\left(\mathbf{n}_{-i}, \mathbf{p}\right)$, $\sigma_{X_{i}}^{2}\left(\mathbf{n}_{-i}, \mathbf{p}_{-i}\right)=\ln M_{i}^{(2)}\left(\mathbf{n}_{-i}, \mathbf{p}\right)-2 \ln M_{i}^{(1)}\left(\mathbf{n}_{-i}, \mathbf{p}\right)$, and
$M_{i}^{(1)}\left(\mathbf{n}_{-i}, \mathbf{p}\right)=\mathbb{E} \ln \left[z_{i} / \operatorname{SINR}_{i}\right]$ and $M_{i}^{(2)}\left(\mathbf{n}_{-i}, \mathbf{p}\right)=$ $\mathbb{E} \ln \left[z_{i}^{2} / \mathrm{SINR}_{i}^{2}\right]$ (see [7] for the derivation of previous statistical expectations).

Expression (2) allows us to resort to the theory of standard interference function [13] [14]. It is possible to show that the following result hold: Let $\mathcal{I}(\mathbf{n}, \mathbf{p})=$ $\left[I_{1}\left(\mathbf{n}_{-1}, \mathbf{p}_{-1}\right), \ldots, I_{i}\left(\mathbf{n}_{-i}, \mathbf{p}_{-i}\right), \ldots, I_{K}\left(\mathbf{n}_{-K}, \mathbf{p}_{-K}\right)\right]^{T}$. For any given rate vector $\mathbf{n}, \mathcal{I}(\mathbf{n}, \mathbf{p})$ is a standard interference function with respect to $\mathbf{p}$, namely:

1) $\mathcal{I}(\mathbf{n}, \mathbf{p}) \succ 0$;

2) if $\mathbf{p} \preceq \tilde{\mathbf{p}}$ then $\mathcal{I}(\mathbf{n}, \mathbf{p}) \preceq \mathcal{I}(\mathbf{n}, \tilde{\mathbf{p}})$;

$3)$ if $c>1$, then $c \mathcal{I}(\mathbf{n}, \mathbf{p}) \succ \mathcal{I}(\mathbf{n}, c \mathbf{p})$.

Furthermore, for any given power vector $\mathbf{p}$, if $\mathbf{n} \preceq \tilde{\mathbf{n}}$ then $\mathcal{I}(\mathbf{n}, \mathbf{p}) \preceq \mathcal{I}(\tilde{\mathbf{n}}, \mathbf{p})$.

Candidate solutions for Problem $\mathcal{P}$ can be found by exploiting the properties of the interference function and a modified optimization problem. A branch-and-bound search reduces efficiently the set of possible solutions, so that the optimal one can be quickly achieved. We approach these issues in the following.

\section{B. Modified problem}

Let us denote with $\mathcal{P}_{1}$ Problem $\mathcal{P}$, where the outage constraint has been approximated with (2):

$$
\begin{array}{llr}
\mathcal{P}_{1}: & \max _{\mathbf{p}, \mathbf{n}} \mathbb{1}^{T} \mathbf{n} \\
\text { s.t. } & p_{i} \geq \gamma_{i} I_{i}\left(\mathbf{n}_{-i}, \mathbf{p}_{-i}\right), & \\
& \mathbf{p}^{T}(\mathbf{n} \circ \mathbb{E}[\mathbf{h}]) \leq P_{T}, & \\
& p_{i 0} \leq p_{i} \leq p_{\max }, & i=1, \ldots, K \\
& 1 \leq n_{i} \leq G_{i 0}, \quad n_{i} \in \mathcal{N} & i=1, \ldots, K
\end{array}
$$

Problem $\mathcal{P}_{1}$ is difficult to solve because the integer rates and the constraint on the total power prevent us to apply iterative algorithms as in [13] and [15]. However, since the rate vector $\mathbf{n}$ belongs to a discrete set, combinatorial optimization techniques can be used for solving Problem $\mathcal{P}_{1}$. With this goal in mind, it can be proved that $(\mathbf{n}, \mathbf{p})$ is the solution of Problem $\mathcal{P}_{1}$ only if the first set of constraints of $\mathcal{P}_{1}$ holds with equality:

Theorem 1: Let $(\mathbf{n}, \mathbf{p})$ be a solution of Problem $\mathcal{P}_{1}$, then:

$$
p_{i}=\gamma_{i} I_{i}\left(\mathbf{n}_{-i}, \mathbf{p}_{-i}\right) \quad \forall i=1, \ldots, K .
$$

Previous theorem allows us to rewrite the outage constraints of Problem $\mathcal{P}_{1}$ at the equality. From this, we propose an approach to solve Problem $\mathcal{P}_{1}$ in two steps. Firstly, we find feasible solutions by a modified problem $\mathcal{P}_{2}$. Secondly, we solve $\mathcal{P}_{1}$ looking at the set of feasible solutions by branchand-bound search.

Feasible rate vectors for Problem $\mathcal{P}_{1}$ can be found by the solutions of the following problem:

$$
\begin{aligned}
\mathcal{P}_{2}: & \min _{\mathbf{p}} \mathbf{p}^{T}(\mathbf{n} \circ \mathbb{E}[\mathbf{h}]) \\
\text { s.t. } & p_{i}=\gamma_{i} I_{i}\left(\mathbf{n}_{-i}, \mathbf{p}_{-i}\right), \quad i=1, \ldots, K
\end{aligned}
$$

Note that in this problem the decision variable is only $\mathbf{p}$, while $\mathbf{n}$, with $n_{i} \in \mathcal{N}$, for $i=1, \ldots, K$, is given. Consider a vector $\mathbf{p}$, given $\mathbf{n}$, that solves $\mathcal{P}_{2}$. This pair of vectors $\mathbf{n}, \mathbf{p}$ provides us with a feasible rate vector for $\mathcal{P}_{1}$ if the constraints $\mathbf{p}^{T}(\mathbf{n} \circ$ 
$\mathbb{E}[\mathbf{h}]) \leq P_{T}$ and $p_{i 0} \leq p_{i} \leq p_{\max }$, for $i=1, \ldots, K$, are satisfied. Therefore $\mathcal{P}_{2}$ is useful, since we can explore the feasibility of a rate vector $\mathbf{n}$ for $\mathcal{P}_{1}$ just by solving Problem $\mathcal{P}_{2}$.

Problem $\mathcal{P}_{2}$ can be solved efficiently by recalling the properties of the interference function. Indeed, these properties allow us to use contraction mappings, which give a low computational cost algorithm that solves Problem $\mathcal{P}_{2}$ by sequences of asynchronous iterations (see [15, Pag. 431]):

$$
p_{i}(t)=I_{i}\left(\mathbf{n}_{-i}, \mathbf{p}_{-i}(t-1)\right) \gamma_{i} \quad \forall i=1, \ldots, K .
$$

The convergence of this algorithm is fast. Monte Carlo simulations show that it takes from 5 to 10 iterations.

One could use (4) to solve Problem $\mathcal{P}_{2}$ for each possible rate vector in $\mathcal{N}$. Thus, the rate vector $\mathbf{n}^{*}$ that solves the Problem $\mathcal{P}_{1}$ could be found using an exhaustive search among all rate vectors in $\mathcal{N}$. However, such a technique has large computational costs, because the cardinality of $\mathcal{N}$ may be very large. In the next subsection, we will present some useful properties of the rate vectors, which, together with a branchand-bound search, allow us to find the optimal solution with reduced computational cost.

\section{Branch-and-bound search}

It is possible to reduce the set $\mathcal{N}$ using local knowledge about specific values of $\mathbf{n}$. In particular, we construct a set of a smaller size over which performing a search for the optimal solution. We propose a technique for the reduction of $\mathcal{N}$ using two criteria. The first one uses the cutting-planes idea [15], whereas the second one is based on considerations about Problem $\mathcal{P}_{1}$. The criteria can be summarized in the following propositions.

Proposition 1: Let $\overline{\mathbf{n}}$ be feasible for $\mathcal{P}_{1}$. A pair $\mathbf{n}^{*}, \mathbf{p}^{*}$ is an optimal solution of $\mathcal{P}_{1}$ only if $\mathbb{1}^{T} \mathbf{n}^{*} \geq \mathbb{1}^{T} \overline{\mathbf{n}}$.

Given a feasible rate vector $\overline{\mathbf{n}}$, from Proposition 1 follows that the search for the optimal solution can be restricted to the set $\mathcal{N}_{\Sigma} \subset \mathcal{N}$, where

$$
\mathcal{N}_{\Sigma}(\overline{\mathbf{n}})=\mathcal{N} \backslash\left\{\mathbf{n} \quad \text { such that } \quad \mathbb{1}^{T} \mathbf{n} \leq \mathbb{1}^{T} \overline{\mathbf{n}}\right\} .
$$

Proposition 2: Let $\overline{\mathbf{n}}$ be unfeasible for $\mathcal{P}_{1}$. If $\mathbf{n} \succeq \overline{\mathbf{n}}$, then $\mathbf{n}$ is unfeasible for $\mathcal{P}_{1}$.

Previous proposition allows us to say that if $\overline{\mathbf{n}}$ is unfeasible, then the set $\mathcal{N}$ can be reduced by defining a set of unfeasible solutions $\mathcal{N}_{I} \subset \mathcal{N}$ according to the following rule:

$$
\mathcal{N}_{I}(\overline{\mathbf{n}})=\mathcal{N} \backslash\{\mathbf{n} \quad \text { such that } \quad \mathbf{n} \succeq \overline{\mathbf{n}}\} .
$$

In practice, Propositions 1 and 2 are useful to reduce the set of feasible rates by using a neural network architecture, where computation and information distribution is optimized. Specifically, a branch-and-bond algorithm can be implemented [16], which allows us to find efficiently the exact solution of Problem $\mathcal{P}_{1}$, since we reduce the set of candidate rate vectors to a smaller one.

\section{Numerical Results}

In this Section we apply our method to a wireless system of third generation. All the system parameters are taken from the 3GPP specifications [17]: the chip time is $T_{c}=2.6 \times$ $10^{-7} \mathrm{~s}$, and the maximum spreading factor is $G_{i 0}=256$. We
TABLE I

SCENARIOS CONSIDERED IN THE NUMERICAL RESULTS.

\begin{tabular}{|c|c|c|}
\hline Scenario & $\sigma_{\xi_{i}}$ & $\alpha_{i}$ \\
\hline \hline $\mathrm{A}$ & $0.5,0.5,0.5,0.5$ & $0.4,0.4,0.4,0.4$ \\
\hline $\mathrm{B}$ & $0.5,0.5,0.5,0.5$ & $0.2,0.2,0.2,0.2$ \\
\hline $\mathrm{C}$ & $0.5,0.5,0.5,0.5$ & $0.7,0.7,0.7,0.7$ \\
\hline $\mathrm{D}$ & $0.4,0.4,0.4,0.4$ & $0.4,0.4,0.4,0.4$ \\
\hline $\mathrm{E}$ & $0.6,0.6,0.6,0.6$ & $0.4,0.4,0.4,0.4$ \\
\hline $\mathrm{M}$ & random in $[0.4,0.6]$ & random in $[0.2,0.7]$ \\
\hline
\end{tabular}

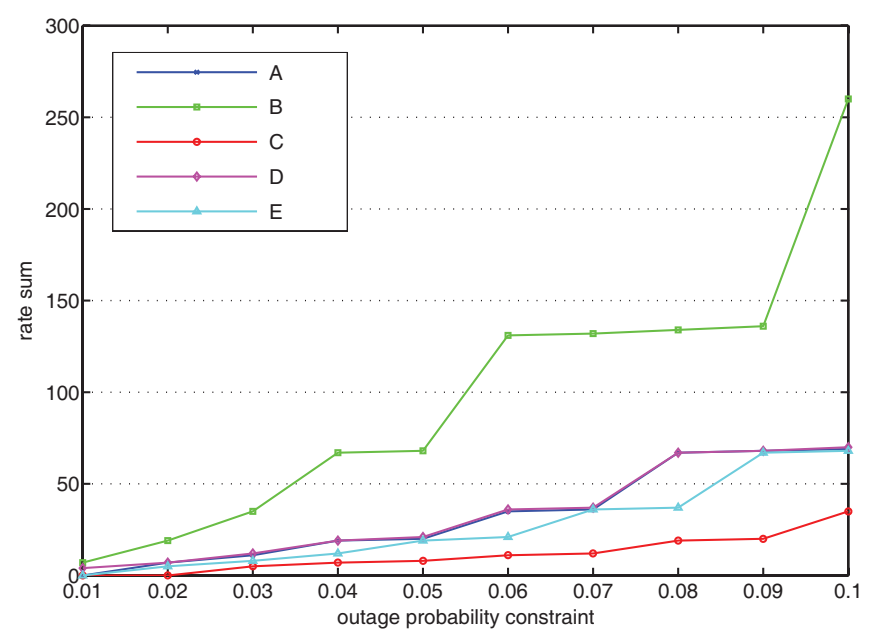

Fig. 1. Optimal solution of Problem $\mathcal{P}_{1}$ as obtained with the branch-andbound algorithm for the cases A, B, C, D and E.

assume a thermal noise $N_{0}=-170 \mathrm{~dB} / \mathrm{Hz}$, and we set $P_{T}=$ $1.5239 \times 10^{-12}$, such that $P_{T} T_{c} / N_{0}=16 \mathrm{~dB}$. We assume a homogeneous propagation environment for the path loss $l_{i}$, which is set to $-90 \mathrm{~dB}$ for each transmitter. As required for the validity of the multiplicative model of the fading, $\mu_{z}=1$ [8]. The system is considered in outage with $\gamma_{i}=3.1$.

We consider the cases of 4,8 and 12 users, for six representative scenarios, denoted with $\mathrm{A}, \mathrm{B}, \mathrm{C}, \mathrm{D}, \mathrm{E}$, and $\mathrm{M}$. In particular, scenarios from $\mathrm{A}$ to $\mathrm{E}$ are referred to the case of 4 users, with uniform standard deviation of the shadowing $\left(\sigma_{\xi_{i}}\right)$ and the source activity $\left(\alpha_{i}\right)$. The sixth scenario considers the case of 4,8 and 12 users with mixed values of $\sigma_{\xi_{i}}$ and $\alpha_{i}$. In Tab. I, the values adopted for $\sigma_{\xi_{i}}$ and $\alpha_{i}$ are reported for each scenario.

\section{A. Rate Maximization}

In Fig. 1 the optimal solution of Problem $\mathcal{P}_{1}$ as obtained with our approach is plotted for the scenarios A to E. Each dot of the curves is referred to a value of the outage probability constraint $O_{i}$ (which is reported on the x axis, and it is assumed to be the same for each user). The maximum throughput achieved is 250 , as obtained for the scenario B when $O_{i}=0.1$. The cost function increases as higher values of the outage constraints are allowed. Observe that better performance is obtained when users have low activity factors $\left(\alpha_{i}=0.2\right.$ in the scenario $\left.\mathrm{B}\right)$, while the minimum rates are achieved when users have high activity $\left(\alpha_{i}=0.7\right.$ in the scenario E). When users have low transmission activity, multi 


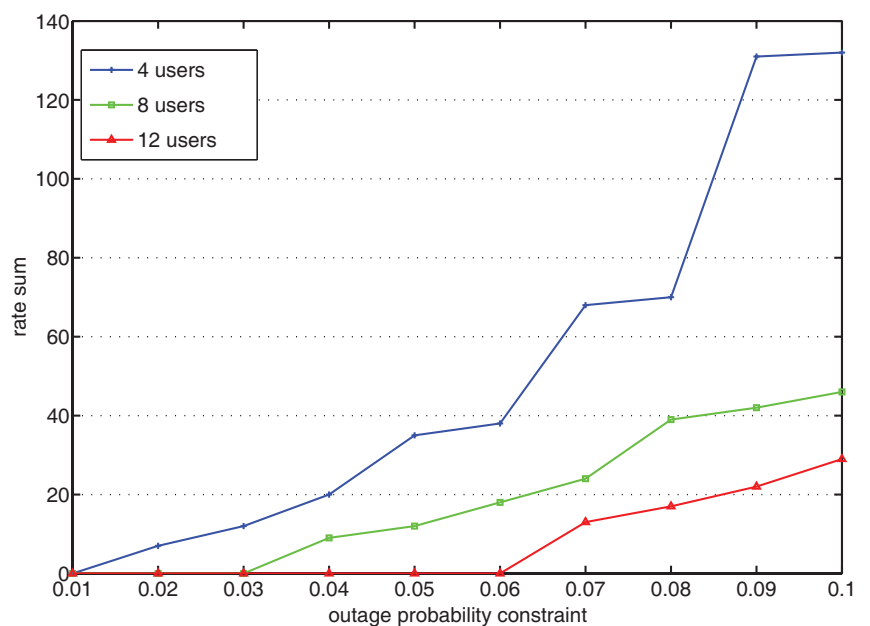

Fig. 2. Optimal solution of Problem $\mathcal{P}_{1}$ as obtained with the branch-andbound algorithm for the scenario $\mathrm{M}$ for 4,8 , and 12 users. Zero rate means that the problem is not feasible.

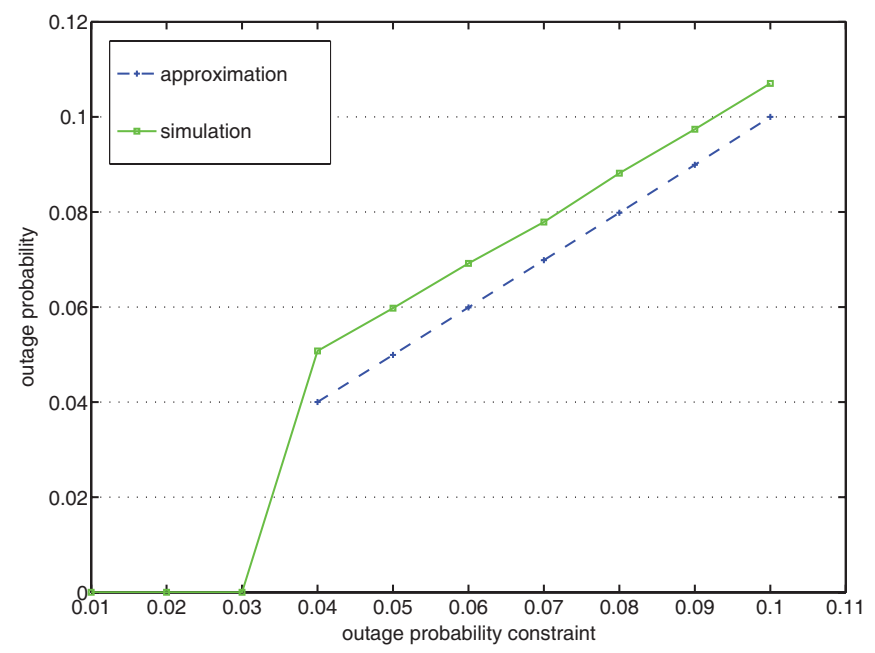

Fig. 3. Outage probability obtained by Monte Carlo simulations (continuous dotted curve). The outage constraint $O_{i}$ on the $\mathrm{x}$-axis is used in the scenario $\mathrm{M}$ for 8 users, where the branch-and-bound algorithm provides us with the optimal rates and powers. These are used to generates samples of SINR, for which the outage probability is plotted on the y-axis.

access interference is reduced, thus enabling higher transmit rates. Also, observe that from the sequence $\mathrm{D}, \mathrm{A}$ and $\mathrm{E}$ as the standard deviations of the shadowing increase, the total rate decreases. The reason is because larger fluctuations of the shadowing increase the multi access interference.

In Fig. 2 the optimal solution of Problem $\mathcal{P}_{1}$ as obtained with our approach is plotted for the scenario $M$. The cases with 4 users feature the highest transmit rates, while the cases with 12 users shows the lowest rates. This is obviously due to the fact that as the number of users increases, the multi access interference is larger. In the case with 12 users, Problem $\mathcal{P}_{1}$ is not feasible for outage probability constraints less than 0.06 , and for outage constraints less than 0.03 for 8 users. This can be seen in the figures where the sum of the rates is zero. The reason of infeasibility is that low outage requirements cannot be guaranteed when there are too many users in the system, namely the number of users exceeds the system capacity [9].
TABLE II

COMPUTATIONAL COMPLEXITY, I.E., NUMBER OF TIMES THAT PROBLEM $\mathcal{P}_{2}$ IS SOLVED.

\begin{tabular}{|l|l|l|l|}
\hline Scenario & $\min$ & $\max$ & average \\
\hline \hline A & 35 & 823 & 335 \\
\hline B & 35 & 2062 & 976 \\
\hline C & 15 & 284 & 105 \\
\hline D & 5 & 895 & 382 \\
\hline E & 15 & 731 & 287 \\
\hline M 4 & 25 & 831 & 335 \\
\hline M 8 & 17 & 20995 & 6073 \\
\hline M 12 & 55 & 22383 & 7131 \\
\hline
\end{tabular}

\section{B. Outage Approximation}

To check the accuracy of the outage approximation adopted in Section III-A, we computed the outage probability corresponding to the rates and powers obtained with the solution of Problem $\mathcal{P}_{1}$ for several cases of the outage requirement. In particular, SINR samples were generated by considering a large set of determinations of the random variables $\xi_{i}$ and $z_{i}$, for $i=1, \ldots, K$, and then the actual outage probability was computed numerically with respect to the threshold $\gamma_{i}$ used in Problem $\mathcal{P}_{1}$. In Fig. 3 we consider the case of 8 users, when they transmit with the rates and powers as obtained by the branch-and-bound algorithm for the scenario $\mathrm{M}$. The outage probability approximation is close to the actual probability. It can be shown that the same behavior is found for all scenarios.

\section{Complexity Analysis}

In this section, we present an analysis of the computational complexity of the branch-and-bound algorithm.

The convergence time of the branch-and-bound algorithm is given by the number of times that Problem $\mathcal{P}_{2}$ must be solved. Proposition 1 and Proposition 2 allow us to reduce the number of candidate solutions with negligible computational complexity. Therefore, the computation cost of the algorithm is given by the computation of the solution of Problem $\mathcal{P}_{2}$. Consequently, we define as computational complexity the number of times for which Problem $\mathcal{P}_{2}$ is solved by the branch-and-bound algorithm.

The computational complexity is evaluated starting the branch-and-bound algorithm with the lowest rate vector. This gives the worst case for the computational complexity, because the active node set is the largest, Proposition 1 and Proposition 2 delete less possible solutions, and hence Problem $\mathcal{P}_{2}$ must be solved more times. In the following, we discuss the computational complexity as obtained by simulations.

In Tab. II, the computational complexity is reported in terms of minimum, maximum, and average number for the scenarios A to $\mathrm{M}$. These figures have been computed averaging over the interval of outage probability requirements. Consider the scenarios $\mathrm{A}$ to $\mathrm{M}$ with 4 users. An exhaustive search over all possible rates would have required to solve Problem $\mathcal{P}_{2}$ for $9^{4}=6561$ times, since there are 9 possible rates per each user. We obtained that the average number of times for which $\mathcal{P}_{2}$ is solved varies from 382 (C) to 976 (B). Observe that the larger 
computational complexity of B is in agreement with the conclusions of the previous Subsection, where it was shown that the scenario B achieves larger sum of the rates. Specifically, starting the branch-and-bound algorithm with the lowest rates, several possible solutions have to be explored before hitting the borders of feasible solutions and finding the optimal one. By the same argument, the scenario $\mathrm{C}$ is the less complex. The average computational complexity for the scenario $M$ with 8 users is 6073 , and with 12 users is 7131 , while it would have been needed to solve Problem $\mathcal{P}_{2}$ a number of times given by $9^{8} \approx 4.30 \times 10^{7}$ and $9^{12} \approx 2.82 \times 10^{11}$, respectively, for an exhaustive search over all the possible rates. We conclude that the good computational complexity of the branch-andbound algorithm is remarkable for all the scenarios we have considered.

\section{CONClusions ANd Future Work}

In this paper, we proposed an approach to allocate the users' powers to maximize the throughput of a CDMA wireless system. A general model of the physical layer was considered, with Rayleigh-lognormal fading and source activity, and quality of service was expressed by the outage probability.

The problem was cast as a mixed integer-real optimization program, where we expressed the constraints by a moment matching approximation of the outage probability. The optimal solution of the problem was derived in two steps: first a modified program was investigated to provide feasible solutions, and then an algorithm, based on branch-and-bound search, was used. Numerical results in scenarios of practical interest confirmed the validity of our approach, and showed that the optimal solution of the problem is achieved with low computational complexity.

\section{REFERENCES}

[1] G. Caire, G. Taricco, and E. Biglieri, "Optimal power control for minimum outage rate in wireless communications," in Proc. IEEE ICC'98, Atlanta, GA, June 1998.
[2] T. Heikkinen, "A model for stochastic power control under log-normal distribution," CWIT 2001, 2001.

[3] S. Kandukuri and S. Boyd, "Optimal power control in interferencelimited fading wireless channels with outage-probability specifications," IEEE Trans. Wireless Commun., vol. 1, pp 46-55, Jan. 2002.

[4] K.-L. Hsiung, S.-J. Kim, and S. Boyd, "Power control in lognormal fading wireless channel with uptime probability specifications via robust geometric programming," ACC, 2005.

[5] J. Papandriopoulos, J. Evans, and S. Dey, "Optimal power control for Rayleigh-faded multiuser systems with outage constraints," IEEE Trans. Wireless Commun., vol. 4, no. 6, Nov. 2005.

[6] — - "Outage-based power control for generalized multiuser fading channels," IEEE Trans. Commun., vol. 54, no. 4, Apr. 2006.

[7] C. Fischione and M. D'Angelo, "An approximation of the outage probability in Rayleigh-lognormal fading scenarios," University of California at Berkeley, http://www.eecs.berkeley.edu/ fischion/papers/outage_approx_report.pdf, Tech. Rep., Mar. 2008.

[8] G. L. Stüber, Principles of Mobile Communication. Kluwer Academic Publishers, 1996.

[9] F. Santucci, G. Durastante, F. Graziosi, and C. Fischione, "Power allocation and control in multimedia CDMA wireless systems," Kluwer Telecommun. Systems, vol. 23, pp. 69-94, May-June 2003.

[10] A. Shah and A. M. Haimovich, "Performance analysis of maximal ratio combining and comparison with optimum combining for mobile radio communications with cochannel interference," IEEE Trans. Veh. Technol., vol. 49, no. 4, pp. 1454-1463, July 2000.

[11] E. Hossain, D. I. Kim, and V. K. Bhargava, "Analysis of TCP performance under joint rate and power adaptation in cellular WCDMA networks," IEEE Trans. Wireless Commun., vol. 3, no. 3, May 2004.

[12] J. S. Evans and D. Everitt, "Effective bandwidth-based admission control for multiservice CDMA cellular networks," IEEE Trans. Veh. Technol., vol. 48, pp. 36-46, Jan. 1999.

[13] J. Herdtner and E. K. P. Chong, "Analysis of a class of distributed asynchronous power control algorithm for cellular wireles system," IEEE J. Select. Areas Commun., vol. 18, no. 3, pp. 436-446, Mar. 2000.

[14] R. D. Yates, "A framework for uplink power contorl in cellular radio system," IEEE J. Select. Areas Commun., vol. 13, Sept. 1995.

[15] D. P Bertsekas and J. N. Tsitsiklis, Parallel and Distribuited Computation: Numerical Methods. Athena Scientific, 1997.

[16] C. Fischione and M. Butussi, "Power and rate control outage based in CDMA wireless networks under MAI and heterogeneous traffic sources," in IEEE International Conference on Communications, June 2007.

[17] 3GPP TS 25.214 V6.1.0, "3rd Generation Partnership Project; Technical Specification Group Radio Access Network; Physical layer procedures (FDD)." 